\title{
PRAXIS EDUCATIVA POR DOCENTES UNIVERSITARIOS PARA UN APRENDIZAJE SIGNIFICATIVO
}

Valles Montero, Karelis del Carmen; Valles de Rojas, Mary Estela; Torres de Nava, Luisenny Marianth; Del Valle Giraldoth, Débora

PRAXIS EDUCATIVA POR DOCENTES UNIVERSITARIOS PARA UN APRENDIZAJE SIGNIFICATIVO

PANORAMA, vol. 15, núm. 29, 2021

Politécnico Grancolombiano, Colombia

Disponible en: https://www.redalyc.org/articulo.oa?id=343967896011

DOI: https://doi.org/10.15765/pnrm.v15i29.2591

\section{(c) (1)(2)}

Esta obra está bajo una Licencia Creative Commons Atribución-NoComercial-Compartirlgual 4.0 Internacional. 
Artículos de investigación científica y tecnológica

PANORAMA, vol. 15, núm. 29, 2021

Politécnico Grancolombiano, Colombia

Recepción: 08 Agosto 2019

Aprobación: 15 Junio 2021

DOI: https://doi.org/10.15765/ pnrm.v15i29.2591

Redalyc: https://www.redalyc.org/ articulo.oa?id=343967896011

\section{PRAXIS EDUCATIVA POR DOCENTES UNIVERSITARIOS PARA UN APRENDIZAJE
SIGNIFICATIVO PARA UN APRENDIZAJE
SIGNIFICATIVO}

Educational praxis by university teachers for meaningful learning

Praxis educacional por professores universitários para uma aprendizagem significativa

Karelis del Carmen Valles Montero karelisvmontero@gmail.com Universidad Nacional Experimental Rafael María Baralt, Venezuela

(1D) https://orcid.org/0000-0003-1256-4164

Mary Estela Valles de Rojas marestroj@hotmail.com Universidad Nacional Experimental Rafael María Baralt, Venezuela

(D) https://orcid.org/0000-0001-7952-2482 Luisenny Marianth Torres de Nava luisennytorres@gmail.com

Universidad Nacional Experimental de la Seguridad, Venezuela

(D) https://orcid.org/0000-0002-3182-8607

Débora Del Valle Giraldoth giraldoth@gmail.com Universidad Nacional Experimental Rafael María Baralt, Venezuela

(1D https://orcid.org/0000-0001-9411-6415

Resumen: El propósito general del presente estudio se orienta a considerar las estrategias de la praxis educativa universitaria, utilizadas por los docentes, en miras de fortalecer el aprendizaje significativo. De igual manera, esta investigación está enmarcada bajo el enfoque cualitativo, de forma amplia naturalista de campo, descriptiva, permitiendo la interpretación, con un método de tipo etnográfico. Para ello se emplearon guías de observación, cuaderno de campo y entrevistas aplicadas a los profesores y estudiantes de educación universitaria. La información que emergió deja en evidencia que es preciso que todo el personal docente en su pleno ejercicio maneje y haga uso de todas las herramientas pedagógicas habidas y por haber dentro del plano educativo para favorecer y lograr con éxito un aprendizaje significativo en los educandos, por lo que todos los docentes deben estar prestos a incorporar y manejar nuevas alternativas y estrategias metodológicas que le permitan logran el objetivo planteado, fortaleciendo tanto la praxis educativa como el aprendizaje significativo.

Palabras clave: Praxis educativa, aprendizaje significativo.

Abstract: The general purpose of this study is oriented to consider the strategies of the university educational praxis, used by teachers, in order to strengthen meaningful learning. Likewise, this research is framed under the qualitative approach, in a broad naturalistic field, descriptive, allowing interpretation, with an ethnographic method. For this purpose, observation guides, field notebook and interviews applied to teachers and students of university education were used. The information that emerged shows 
that it is necessary that all teachers, in their full exercise, handle and make use of all the pedagogical tools available in the educational field to favor and successfully achieve significant learning in students, so that all teachers must be ready to incorporate and handle new alternatives and methodological strategies that allow them to achieve the proposed objective, strengthening both educational praxis and significant learning.

Keywords: Educational praxis, meaningful learning.

Resumo: O objectivo geral deste estudo está orientado para considerar as estratégias da práxis educacional universitária, utilizadas pelos professores, a fim de reforçar a aprendizagem significativa. Do mesmo modo, esta investigação é enquadrada sob uma abordagem qualitativa, num vasto campo naturalista, descritiva, permitindo a interpretação, com um método etnográfico. Para este fim, foram utilizados guias de observação, cadernos de notas de campo e entrevistas com professores e estudantes do ensino universitário. A informação que surgiu torna claro que é necessário que todo o pessoal docente, no seu exercício pleno, trate e faça uso de todas as ferramentas pedagógicas disponíveis e que se encontrem dentro do campo educacional para promover e alcançar com sucesso uma aprendizagem significativa nos estudantes, pelo que todos os professores devem estar prontos para incorporar e gerir novas alternativas e estratégias metodológicas que lhes permitam alcançar o objectivo declarado, reforçando tanto a práxis educacional como a aprendizagem significativa.

Palavras-chave: Praxis educativa, aprendizagem significativa.

\section{INTRODUCCIÓN}

La educación superior venezolana enfrenta el desafío de los cambios en la derivación de una actitud crítica del docente formador de formadores, desde su propia praxis educativa para generar nuevas perspectivas de accionamiento y competencias docentes interpretadas en el ámbito del aprendizaje significativo. Desde este enfoque, el razonamiento se articula con la formación integral de los futuros docentes para que participen en los procesos de transformación que requiere el país, lo cual significa su presencia protagónica como actor clave en la propia experiencia didáctica, basada en la reflexión crítica y generativa de su formación educativa, con la plataforma sustantiva en el conocimiento y advenimientos de variadas exigencias curriculares.

En tal sentido, el docente en cumplimiento de su rol dentro del aula debe estar sujeto a la planificación, tomando en consideración el desarrollo de los procesos cognoscitivos y afectivos del estudiante, así como la participación activa que deben tener en la construcción de su propio aprendizaje (Ruiz-Bolívar, 1998).

En función a lo antes señalado, la presente investigación sobre las praxis educativas para un aprendizaje significativo pretende mostrar, con un lenguaje sencillo, las actividades, procedimientos, encaminados hacia una labor docente eficiente y eficaz; además, estimular a los actores del proceso universitario hacia una formación basada en el desarrollo de praxis educativa para el aprendizaje significativo por docentes universitarios.

Igualmente, bajo esta perspectiva, esta investigación está orientada a considerar las estrategias de la praxis educativa universitaria, utilizadas por los docentes en miras de fortalecer el aprendizaje significativo; estudio que se llevó a cabo dentro del enfoque cualitativo mediante el método etnográfico. 


\section{Praxis educativa}

En la praxis educativa, la reflexión y la acción del docente in situ generan cambios y transformaciones. El ámbito universitario es propicio, ya que vincula aspectos científicos, técnicos y del quehacer cotidiano que exigen reflexión, creación y transformación permanente. Referente a esto, Delgado (2017), afirma:

"La praxis es una práctica social que se concibe como un conjunto de actuaciones de los actores sociales, con las que se pretende satisfacer sus necesidades y que implica unas acciones operativas, de actitudes y comportamientos” (p.21).

Así mismo, Freire (2018) señala:

La praxis educativa es un reflexionar de manera crítica sobre la práctica docente, y sobre la manera de actuar con los educandos. El supuesto fundamental que subyace a esta visión es el imperante deber del profesor de respetar la dignidad del educando, su autonomía, su identidad en proceso. Es necesario que los profesores reflexionen sobre su práctica pedagógica de modo permanente, en el marco de un análisis social, político, económico, cultural más amplio. Ello implica que la competencia pedagógica del educador no se restringe a sus conocimientos teóricos disciplinares y didácticos, sino que, además, exige tener una visión y postura frente al mundo y frente al contexto real en el que se está dando la acción educativa. (p.30)

Por consiguiente, la práctica educativa o praxis educativa se puede definir como el conjunto de vivencias o situaciones enmarcadas dentro del marco institucional que influye de manera positiva en el proceso de enseñanza y aprendizaje, una vez que las interacciones ente profesores y estudiantes se vinculen con las realidades y con el entorno que les rodean, y no solo sujetas a un aula de clases, lo que en gran medida está determinado por las lógicas de gestión y organización institucional del centro educativo (García-Cabrero et al., 2008).

\section{Aprendizaje significativo}

El aprendizaje significativo va de la mano con un cambio en el significado de las experiencias. En este sentido, en la experiencia humana entran en juego el pensamiento y la afectividad; una vez que se consideren y se conjuguen estos elementos se capacita al individuo y, por ende, se logra en él un enriquecimiento significativo de sus experiencias (Ausubel, 2018).

En el ejercicio docente, para lograr un aprendizaje significativo se deben considerar varios elementos tales como los didácticos, estructuras lógicas, estructuras psicológicas, experiencias previas, entre otras, que fortalezcan y retroalimenten la formación y las experiencias cognitivas del estudiante(Acevedo-Valencia \& Suárez-Saldaña, 2015; Díaz, 2016; Rivera \& Coronado, 2015)(Guzmán, 2015; Rivera \& Coronado, 2015; Vargas, Pichardo, \& Íñiguez, 2015).

En tal sentido, es menester que los docentes didácticamente exploren los conocimientos previos de los estudiantes, ya que estos son la 
base fundamental para lograr el aprendizaje significativo; esto ocurre cuando una nueva información se conecta con un concepto relevante persistente en la estructura cognitiva, que tendrá implicaciones en las nuevas ideas conceptuales del alumno, donde estos podrán aprender significativamente en la medida en que estén claramente disponibles en la estructura cognitiva y funcionen como punto de anclaje de los conocimientos iniciales concebidos con los nuevos (Hernández, 2017).

En ese orden de ideas, y entendiendo que el objetivo principal de la labor educativa es lograr un aprendizaje significativo en el estudiante, él mismo debe ser capaz de contribuir significativamente con ello, cultivando constructivamente su memoria comprensiva, es decir, que tenga la fortaleza de aprender a aprender, por medio de la interaccion activa docente- estudiante y de los estudiantes entre sí (Garza, 2018).

\section{Enfoque epistemológico}

El presente estudio se circunscribe en un enfoque paradigmático cualitativo, centrado en la metodología etnográfica interpretativa, basada fundamentalmente en la recolección de datos, los cuales muestran un hecho, fenómeno o situación ocurrida. Al respecto, Sandín (2005) manifiesta: "La investigación cualitativa se refiere a la vida de las personas, historias, comportamientos, y también el funcionamiento organizativo, movimientos sociales, relaciones o interacciones; dichos datos pueden ser cuantificados, pero el análisis en sí mismo es cualitativo" (p.47).

Ahora bien, la investigación está orientada a considerar las estrategias de la praxis educativa universitaria, utilizadas por los docentes, en miras de fortalecer el aprendizaje significativo. En esta indagación se necesita la recolección de datos y aplicar un proceso de interpretación, que es lo que permite obtener el criterio u opinión de cada uno de los participantes fundamentándose en las características de dicho enfoque; por esta razón se selecciona el paradigma de investigación cualitativo, porque este permite conocer las inquietudes de los docentes y los estudiantes, sus intereses, necesidades, problemas y expectativas. Por otra parte, permite interactuar con estos sujetos en su ambiente natural, es decir, en su contexto (dentro de la institución o aula de clase).

\section{MÉTODO}

\section{Método de investigación}

El método empleado en la investigación es el etnográfico interpretativo, puesto que tal procedimiento aporta estrategias, técnicas y recursos que pueden ser aplicados y desarrollados directamente en la unidad de análisis, es decir, en el contexto natural donde se desarrollan los acontecimientos, hechos o fenómenos observados sin falsear la realidad. 


\section{Diseño de la investigación}

El esquema tomado como referencia para este estudio es el planteado por Bonilla y Rodríguez (2017, p.127), el cual consta de tres momentos: definición de la situación problema, trabajo de campo e identificación de patrones culturales. A consideración de las autoras, el nuevo diseño comprende cinco momentos, readaptados y denominados de manera distinta, pero que en la práctica inducen a la misma esencia de toda investigación científica; estos constan de: descripción de la temática de estudio, aproximación teórica, momento metodológico, momento de análisis de contenido, categorización y teorización y, finalmente, hallazgos de la investigación. Igualmente, el diseño está subdividido en varios segmentos que explicitan el contenido de cada momento (Alzate \& Góez, 2017; León, 2014).

A continuación, se presenta un diagrama con las adaptaciones realizadas por las investigadoras, donde se resume el proceso utilizado para este estudio:

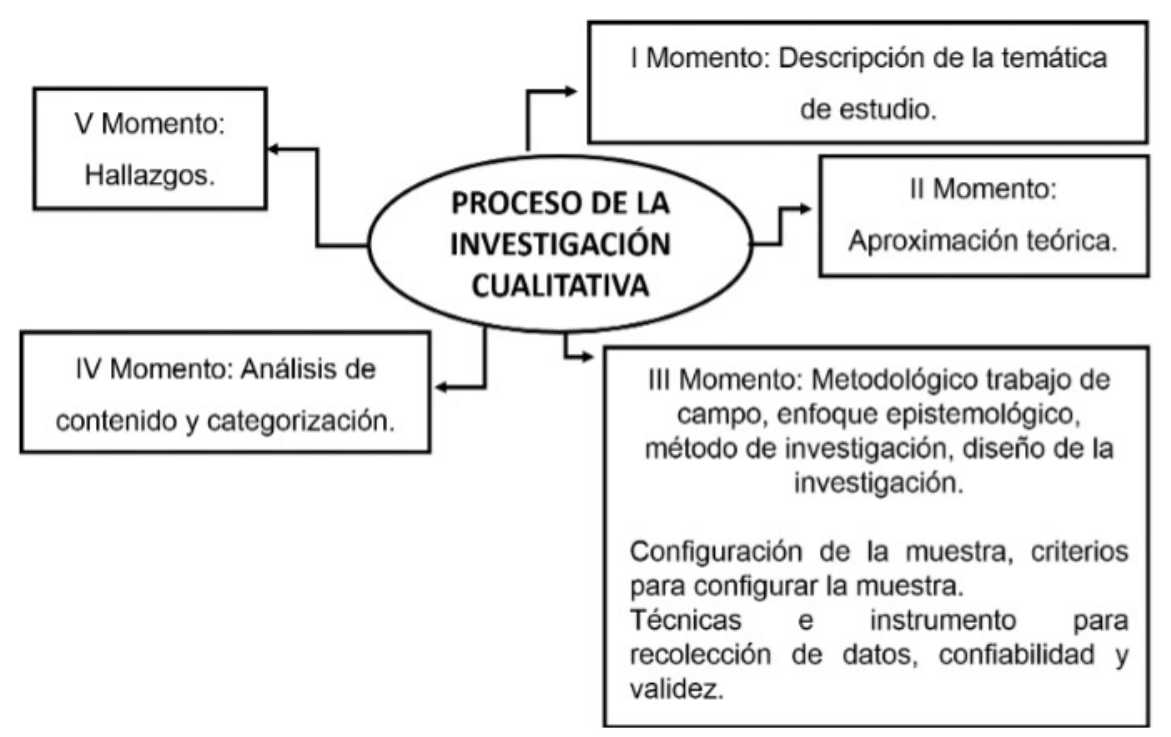

\section{Diagrama 1.}

El proceso de la investigación cualitativa.

Elaboración propia (2021), con base en Bonilla y Rodríguez (2017).

El tipo de diseño aplicado para el estudio se ubica dentro del enfoque introspectivo vivencial, específicamente en la tradición cualitativa etnográfica. De allí que al tomar en consideración el recorrido epistemológico o secuencia operativa realizada durante el proceso de indagación, obtención de la información, interacción con los sujetos observados, puesta en práctica de técnicas e instrumentos de recolección de datos, quedó estructurado de la siguiente manera:

Configuración de la muestra: en las investigaciones cualitativas la muestra no se selecciona, se configura de acuerdo con los propósitos que se haya planteado el investigador para comprender el fenómeno que estudia. Para los efectos de este estudio se establecen una serie 
de criterios para configurar la muestra, considerando el contexto de educación universitaria.

Criterios para configurar la muestra: para la elaboración de los criterios se consideró la experiencia previa como investigadores, así como los contactos que se tuvo durante la etapa de pesquisa en el escenario de investigación; a continuación, se presentan los criterios bajo los cuales queda configurada la muestra: personal docente y estudiantes.

\section{Técnicas e instrumentos para recolectar los datos}

La técnica utilizada en esta investigación fue la observación participante, y como instrumento para la recolección de los datos se empleó la entrevista en profundidad. La primera permitió conocer la realidad de los estudiantes en el campo universitario, en el que se formó parte del grupo sin la intervención directa, y en la segunda se estableció una conversación amena y poco formal, tanto con los docentes como con los estudiantes universitarios, donde pudieron expresar abiertamente sus ideas, pensamientos y sentimientos. Para recabar la información durante la entrevista se utilizó un guion de entrevista contentivo de algunas interrogantes dirigidas a los informantes claves, de manera abierta y flexible según su opinión y criterio personal(Montes, 2015).

\section{Confrabilidad y validez}

Se realizó la triangulación como método de contraste de las informaciones obtenidas por medio de las observaciones y entrevistas. Para Kemmis, citado por Pérez (1994, p.81), la triangulación "...consiste en un control cruzado entre diferentes fuentes de datos: personas, instrumentos, documentos o la combinación de estos".

En referencia a lo expresado anteriormente, la triangulación permitirá el control en la coherencia de los diferentes relatos y obtener una comprensión profunda y clara de la realidad estudiada. Tomando como base los tipos de triangulación planteadas por Pérez (1994, p.85), en este trabajo se realizó una triangulación que consistió en utilizar el mismo método en diferentes ocasiones y cruzar las informaciones obtenidas por las diversas fuentes: la observación participante y las entrevistas en profundidad.

En este marco de ideas, la triangulación hizo posible el establecimiento de comparaciones que permitieron actuar como filtros mediante los cuales se pudo captar de modo selectivo los elementos, estrategias y procesos de la educación a nivel universitario. Procesos que coincidirán en las diferentes fuentes, permitiendo evidenciar la validez del estudio. 


\section{RESULTADOS}

\section{Análisis de la información y categorización}

En relación con la praxis educativa por docentes universitarios para un aprendizaje significativo, partiendo de las premisas que se lograron establecer con las entrevistas realizadas, se desprendieron cuatro categorías, entre las que se destacan: Estrategias educativas aplicadas en el desarrollo de saberes, Estrategias metodológicas utilizadas para el aprendizaje de los estudiantes, Incorporación de la praxis educativa en el desempeño académico y, por último, Reflexión crítica de la praxis educativa como profesor universitario, cada una de ellas descrita a continuación.

\section{Estrategias educativas aplicadas a la praxis en el desarrollo de saberes}

Esta categoría se presenta dentro de esta investigación como el apoyo que el docente les ofrece a sus estudiantes antes, durante y al culminar la ejecución de sus prácticas. Es decir, brindándole la información necesaria para programar y llevar cabo cada una de las actividades exigidas dentro de estas asignaturas, que hagan posible que puedan ser desarrolladas con el mayor éxito posible.

Cabe señalar que los sujetos en estudio señalaron que ellos utilizaban:

Las lecturas comprensivas, cuadros sinópticos, mapas conceptuales, la construcción colectiva del saber a través de los programas integrales de formación, tanto virtual como presencial. Otro sujeto manifestó que él utilizaba la educación popular, como el intercambio de ideas y experiencias, los videoforos, las conferencias. Aunado a esto, otro señaló que las estrategias utilizadas en los ambientes de clases los llevan a encontrarse en una sociedad del conocimiento en la cual deben y tienen esta enorme responsabilidad de realizar el proceso de enseñanzaaprendizaje, aprendizaje- enseñanza; la retroalimentación se con el uso de las nuevas tecnologías como soporte de aprendizaje.

De igual modo, esta situación se hizo notoria en las observaciones realizadas, ya que se evidencia que sí se están aplicando, pero en una minoría, que existen muchos profesores que todavía se niegan a los cambios emanados por los entes educativos. La docencia universitaria implica el abordaje y la vinculación de aspectos científicos, técnicos así como del quehacer cotidiano, lo que favorece la crítica, la reflexión, la creación y la transformación.

En función a lo antes mencionado, el docente debe estar abierto a los cambios y trasformaciones sociales a favor de lograr un aprendizaje significativo en los educandos, por ende debe estar sujeto a los cambios que favorezcan la actualización en pro de su praxis educativa. En ese mismo orden de ideas, Freire (2018) afirma que es necesario que los docentes replanteen sus prácticas pedagógicas de manera continua, aborden y profundicen el análisis de tipo social, político, económico y cultural, sin 
dejar de respetar el criterio del educando, su autonomía y su identidad en el proceso.

$\mathrm{Al}$ respecto, Zavalza (2006) refiere que en la práctica educativa como proceso reflexivo y dinámico debe estar inmersa la pedagogía antes y después de los procesos interactivos en el aula. Estos comprenden la interacción entre docente-estudiante y estudianteestudiante. Igualmente, el docente debe continuamente actualizar sus conocimientos, innovar estrategias, desarrollar habilidades y actitudes, aplicar acciones fundamentadas en teorías educativas que, desde el punto de vista cognitivo, afectivo, social y psicomotor, permiten entender y atender al estudiante.

\section{Estrategias metodológicas utilizadas para el aprendizaje de los estudiantes}

Esta categoría está vinculada con las estrategias que debe utilizar el profesor para que lo conlleve a aplicar aprendizajes significativos con sus alumnos. Es decir, preparar al estudiante para que pueda desarrollar sus conocimientos, habilidades dentro del entorno donde se desenvolverán.

$\mathrm{Al}$ respecto, los sujetos en estudio coincidieron en afirmar que ellos implementaban talleres, debates, intercambios de saberes por medio del diálogo reflexivo, mesas de trabajo, exposiciones y también en la formación de preguntas, grupos de trabajos y el diálogo. Por su parte, otros sujetos opinaron que utilizaban la elaboración de los proyectos de vida, las reflexiones y el IAP según la unidad curricular, como también la contextualización de los contenidos con la realidad social, política, económica y cultural e histórica de la patria. Algunos docentes manifestaron que la planificación es de suma importancia para que conlleve a un aprendizaje significativo: planificar, investigar, para poder llegar al éxito de ese aprendizaje y que le quede a los estudiantes que participan en las clases.

En función a lo antes señalado, Díaz y Hernández (2018) plantean que para que un aprendizaje resulte significativo se deben tomar en cuenta varios elementos; uno de ellos es el manejo de la nueva información, la cual debe tener una relación de modo no arbitrario y sustancial con lo que el estudiante ya conoce, es decir, tomar en consideración sus experiencias previas, así como la disposición del estudiante. Finalmente, evaluar los contenidos de la materia, así como el ambiente donde se desarrolle o se vaya a desarrollar el aprendizaje.

Es propicio mencionar que para que el aprendizaje significativo se pueda lograr, el material de aprendizaje presentado por el docente debe ser potencialmente innovador y, por ende, significativo, considerando la estructura lógica de la materia o área de conocimiento y la estructura psicológica del estudiante. Que estos recursos sean capaces de promover un conflicto cognitivo que provoque la necesidad de modificar los esquemas mentales preestablecidos, proveyendo información que le ayude a reestructurar esos esquemas mentales, que es lo que el docente premeditadamente trata de romper, logrando de esta manera que el aprendizaje resulte significativo (Díaz \& Hernández, 2018). 
En tal sentido, las estrategias que debe utilizar el profesor para lograr un aprendizaje significativo en sus alumnos, deben estar enmarcadas en la creación de un entorno de instrucción, basado en el entendimiento y la comprensión, más que en la memorización; en función a ello, los alumnos deben ser capaces de aplicar este conocimiento en diferentes contextos y situaciones o realidades de su día a día, utilizando asertivamente las herramientas de aprendizajes adquiridas. No obstante, como lo ha señalado Díaz y Hernández (2018), la motivación del estudiante es fundamental, y para ello el docente debe hacer uso de sus habilidades pedagógicas para lograr en los educandos un aprendizaje significativo.

En concordancia con lo antes señalado, se pudo observar que algunos profesores, en miras de lograr un aprendizaje significativo, implementan diferentes estrategias partiendo de los conocimientos previos que traen los estudiantes, favoreciendo el intercambio de saberes utilizando el diálogo reflexivo, las mesas de trabajos y exposiciones, así como la realización de talleres, debates y videoforos, considerando la planificación y la investigación para reforzar y fortalecer el aprendizaje y adquisición de nuevos conocimientos.

Considerando las teorías planteadas acerca del logro del aprendizaje significativo en los estudiantes, los docentes deben estar listos ante los nuevos cambios y trasformaciones que posibiliten las herramientas para lograr la significancia del aprendizaje; en tal sentido, todos los docentes deben involucrarse con la realidad social, contextualizar los contenidos y valorar la retroalimentación con respecto a las experiencias previas de los estudiantes, para así adecuar las mejores estrategias de aprendizaje que permitan reordenar y organizar las estructuras cognitivas adquiridas por los estudiantes, garantizando una formación de calidad, como lo establece la ley.

\section{Incorporación de la praxis educativa en el desempeño académico}

Esta categoría parte de la pertinencia que debe surgir para que el aprendizaje significativo aplicado a los estudiantes por sus profesores se pueda cumplir. En las entrevistas surgieron los siguientes hallazgos y puntos de vista.

Se incorpora a través de un diagnóstico que se realiza por parte del docente, luego se socializa las estrategias seleccionadas con los estudiantes y se escuchan los aportes que los mismos puedan dar a los docentes, ya que para un aprendizaje significativo es necesario prestar atención a las necesidades educativas y cómo aprenden los estudiantes.

El saber se amplía con otros colectivos estudiantiles por medio de mesas de trabajos y proyectos. Se trata de un proceso dialéctico y organizado por momentos de aprendizaje de forma progresiva y constante; todos los días se aprende algo nuevo como profesor y como estudiante.

Con relación a lo observado se evidenció que los profesores sujetos de estudio diagnosticaron, luego socializaron las estrategias seleccionadas con los estudiantes y se escucharon los aportes que los mismos pudieron dar al profesor. De igual forma, se observó que el profesor entiende que 
para un aprendizaje sea significativo es necesario prestar atención a las necesidades educativas y a cómo aprenden los estudiantes, lo cual se puede lograr mediante la investigación formal y la retroalimentación con ellos mismos, también por medio de charlas interactivas en internet. Se demostró compromiso con la dinámica educativa dentro del ambiente de clase, con métodos de reflexión, métodos prácticos y dinámicos.

El aprendizaje significativo conduce a un cambio en el significado de la experiencia. En este sentido, la experiencia humana no solo implica pensamiento, sino también afectividad y únicamente cuando se consideran en conjunto se capacita al individuo para enriquecer el significado de su experiencia. (Ausubel, 2018, p.61)

Ausubel (2018) también señala que el aprendizaje del estudiante depende de las experiencias previas, es decir, de la estructura cognitiva preestablecida que se relaciona con la nueva información, entendiendo que la estructura cognitiva involucra conceptos e ideas que un individuo posee y maneja en un determinado campo del conocimiento. Para el autor, es de vital importancia conocer la estructura cognitiva del estudiante, resaltando que no solo se trata de saber la cantidad de información que posee, sino cuáles son los conceptos y proposiciones que maneja, así como su grado de estabilidad.

En relación con lo antes señalado, es oportuno destacar que para lograr un aprendizaje significativo, el docente debe planificar actividades que les sean familiares a los estudiantes, pero también hay que enfrentarse a una serie de conocimientos previos que pueden ser distintos, y encontrarse a la vez con el estudiante que no posee antecedentes sobre lo que se pretende enseñar; cuando esto ocurre es factible que el docente utilice habilidades generadoras de información previas, como estrategias que le permitan a el estudiante activar, relacionar y compartir conocimientos anteriores sobre un tema determinado.

Es propicio destacar que una de las metas u objetivos que persigue el docente para lograr un aprendizaje significativo en sus estudiantes es el de incentivar la capacidad para que estos puedan enriquecer sus conocimientos por sí solos y que a su vez, al asociar y relacionar los conocimientos previos con la nueva información, puedan alcanzar ese aprendizaje significativo.

Esto se relaciona con lo señalado por AMEI (2019), que resalta que la intervención educativa debe tener como objetivo prioritario el posibilitar que los estudiantes realicen su propio aprendizaje significativo, considerando la interactividad docente - estudiante o estudiantes entre sí, cultivando de manera constructiva su memoria comprensiva, enriqueciendo su estructura cognitiva, por lo que se hace fácil lograr su propio aprendizaje, logrando aprender a aprender.

\section{Reflexión critica de la praxis educativa como profesor universitario}

Esta categoría surge dentro de la formación que todo profesor universitario debe tener, como todos aquellos conocimientos y competencias adquiridas durante el desarrollo de la carrera y que 
le permiten ser aplicados durante el desarrollo de su praxis como profesional. Desde esta perspectiva, según los testimonios de los sujetos entrevistados, se obtuvieron los siguientes resultados.

Se ha podido evidenciar con la praxis empleada que es un poco difícil que pueda emerger en los ambientes de clase el despertar en los estudiantes sobre el aprendizaje significativo, debido que se observa que los alumnos poco leen y se ilustran con respecto a los temas dados, por lo tanto, para llegar al objetivo inicial del pensamiento crítico reflexivo hay que esforzarse bastante.

El tema de la praxis educativa se debe llevar muy cuidadosamente, pues no se trata simplemente de decir algo y hacerlo, sino vivirlo y creerlo; por tanto, no es un asunto que se da de la noche a la mañana, sino que exige un proceso en el que se va descubriendo a medida que los sujetos se involucran con lo que hacen. Por otra parte, se debe estar siempre comprenetrado con lo que está viviendo la sociedad, es decir, no vivir aislado de la realidad. En este sentido, lo ético- político debe estar inmerso con lo que se hace en los salones de clases.

De igual manera, los docentes deben permanecer continuamente actualizados en los acontecimientos en pleno desarrollo. De lo contrario, solo estarían depositando contenidos programáticos en los estudiantes, sin desarrollar el pensamiento crítico, que es clave en la educación popular.

Ser docente universitario implica una responsabilidad muy grande, partiendo del hecho de que la concepción misma de la docencia implica cambios y trasformación, es decir, la educación de por sí es un proceso trasformador, lo que implica que cada docente como tal, en su día a día, debe estar en un continuo aprendizaje y aceptar las inserciones de las nuevas tecnologías con el propósito de extraer lo mejor de ellas para el beneficio de la enseñanza y aprendizaje.

Como se ha dicho, es importante reiterar la necesidad de estimular la acción hacia la crítica reflexiva, partiendo de la realidad del entorno que le rodea, de las situaciones actuales, por lo que es imperante contextualizar los contenidos programáticos, llevarlos a la realidad social, con el fin último de tratar de propiciar cambios en el pensar y accionar sus estudiantes en beneficio de la sociedad.

Si bien es cierto que la educación conductista ha jugado -y quizás aún juega- un papel importante dentro de la formación del estudiante universitario, precisamente es aquí donde el docente debe reestructurar su modelo pedagógico y estimular el aprendizaje significativo por medio del constructivismo, hacer del estudiante protagonista de su propio conocimiento y, por ende, de su propio aprendizaje significativo.

En ese mismo orden de ideas, Olaves (2018) señala que es preciso que el docente internalice la educación como un proceso transformador y dinámico, centrado en la construcción del conocimiento partiendo de las vivencias del día a día, enlazándolas de manera creativa con la información. Esto propicia la construcción de los saberes de los alumnos de forma activa, considerando que enseñar es facilitar herramientas, lejos de la simple memorización o la repetición y más cercano a la creación y la construcción de su propio aprendizaje. 
Sumado a lo antes señalado, Aldape (2018) afirma que los nuevos paradigmas educativos y pedagógicos, fundamentados en los constructos de la psicología y de la ciencia cognitiva sobre cómo aprende el ser humano, conducen a reconocer que el estudiante debe aprender estrategias cognitivas, es decir, considerar procedimientos para adquirir, recuperar y aplicar la información. En ese orden de ideas, el constructivismo sociocultural, precisamente, sitúa la actividad mental del educando en la base de la apropiación del conocimiento, partiendo del hecho de que un conocimiento se apropia cuando se interioriza y se incorpora a la estructura mental.

Con lo expuesto, Abelló (2018) deja en evidencia los diferentes e importantes roles que juega el docente en el aprendizaje del educando, entre lo que se destaca la participación como instrumento mediador del encuentro del alumno con el conocimiento, como facilitador de las condiciones para la construcción del conocimiento y como inspirador y/o creador para generar transformación, a sabiendas que el proceso de enseñanza-aprendizaje es considerado una unidad pedagógica compartida y creativa.

En función de lo antes señalado, es propicio resaltar que el docente debe ser capaz de manejar una serie de herramientas (teóricas, conceptuales, procedimentales y metodológicas), que le permitan conocer a profundidad el entorno y a sus educandos. En tal sentido, como profesional en la docencia, es imperante dominar el campo del conocimiento específico y tener elementos que le permitan profundizarlos, aplicarlos y estar en permanente actualización (Hortal, 2018).

Hoy día, el docente debe estar familiarizado con los nuevos paradigmas educativos, comprender que su rol va más allá que el simple catedrático tradicionalista, el cual induce un aprendizaje repetitivo y memorístico en sus alumnos. El docente debe estar centrado en crear e innovar, así como facilitar la construcción del conocimiento en sus educandos, mediante cada experiencia vivida y, sobretodo, motivar la autoformación (Aldape, 2018).

Es debido destacar que, de manera general, el docente universitario ha de contar con elementos y herramientas que le brinden la oportunidad a sus educandos para que ellos mismos sean capaces de construir y poner en práctica sus propios instrumentos de aprendizaje. En ese sentido, Colomer (2018) señala que el profesor universitario es considerado en esencia como un innovador de métodos y de ambientes de aprendizaje, capaz de formar equipos de trabajo junto con sus alumnos y con otros profesores, con la tendencia de desaprender para aprender, para descubrir, innovar y difundir así el conocimiento.

\section{DISCUSIÓN Y CONCLUSIÓN}

El docente debe mantenerse en constante actualización, fortaleciendo sus conocimientos, habilidades y actitudes, imponiendo acciones fundamentadas en teorías educativas que, desde el punto de vista 
cognoscitivo, afectivo, social y psicomotor, permiten entender y atender al estudiante.

Dentro del amplio universo de enseñar es imperante que el docente brinde y maneje tanto estrategias como herramientas pedagógicas necesarias a favor del aprendizaje significativo, pero para ello es menester conocer los estudiantes y adecuar tales estrategias y herramientas a las realidades de los mismos y del entorno que les rodea.

Es relevante que las clases sean de naturaleza enteramente participativa, creativa y transformadora. En función a ello, es preciso establecer una interacción activa y dinámica entre profesor- alumno y alumno- profesor, así como estudiantes entre sí, lo que contribuiría significativamente con la calidad de la docencia misma y, por ende, con el aprendizaje significativo.

En consideración a la incorporación y manejo de las nuevas alternativas de estrategias metodológicas por parte del docente -a favor de enriquecer la praxis educativa y, por ende, el aprendizaje significativo en pro de los estudiantes- el profesor debe propiciar a los estudiantes a que incorporen en los aprendizajes sus experiencias vividas, que sean capaces de asociarlas y relacionarlas, que a su vez estos sean capaces de aprender a aprender, para que su aprendizaje sea continuo y permanente.

Dentro del desarrollo de la praxis educativa, el docente universitario -quien ejerce un rol como instrumento mediador y facilitador del conocimiento-, debe mantener el propósito de conducir en los estudiantes a construir su propia formación, conocimiento y aprendizaje, es decir, que el estudiante se responsabilice de orientar y manejar su propia información.

\section{REFERENCIAS BIBLIOGRÁFICAS}

Acevedo-Valencia, L., \& Suárez-Saldaña, J. E. (2015). Los operadores económicos autorizados en Colombia: :puede el modelo estandarizado de la Organización Mundial de Aduanas facilitar la inserción nacional en la realidad del comercio exterior mundial? Panorama, 9(17), 124-131. http s://doi.org/10.15765/pnrm.v9i17.796

Abelló, L. (2018). El desarrollo de competencias docentes en la formación del profesorado. España: Secretaría General Técnica. Ministerio de Educación y Ciencias.

Aldape, T. (2018). Desarrollo de las competencias del docente. Demanda de la Aldea Global del siglo XXI. México: Editorial Libros en Red.

AMEI. (2019). XXXIII Congreso Anual de la AMEI - Monterrey.

Ausubel, D. (2018). Psicología educativa: un punto de vista cognoscitivo. México: Editorial Trillas S.A.

Alzate, D. M. O., \& Góez, Á. M. B. (2017). LA NUEVA MISIÓN DE LA UNIVERSIDAD. CONTEXTUALIZACIÓN Y RESULTADOS: CASOS DE TRES UNIVERSIDADES PÚBLICAS COLOMBIANASLA NUEVA MISIÓN DE LA UNIVERSIDAD. CONTEXTUALIZACIÓN Y RESULTADOS: CASOS DE TRES UNIVERSIDADES PÚBLICAS COLOMBIANAS-The new mission of... Panorama, 11(20), 82-94. https://doi.org/10.15765/pnrm.v11i20.1 010 
Bonilla, E., \& Rodríguez, P. (2017). Más allá del dilema de los métodos. La investigación en las ciencias sociales. Bogotá: Ediciones Uniandes.

Colomer, A. (2018). La participación en las administraciones públicas: ¿cooperación o enfrentamiento? España: UPV.

Correa, M. (2006). Contexto, interacción y conocimiento en el aula. Revista Pensamiento Psicológico, 2(7), 133-148.

Delgado, F. (2017). El pluralismo axiológico de la ciencia y praxis educativa. Madrid: Ediciones Alfaguara.

Díaz, F., \& Hernández, G. (2018), Estrategias docentes para un aprendizaje significativo. Una interpretación constructivista. Ciudad de México, México: McGraw Hill Interamericana S.A.

Díaz, A. M. (2016). Evaluación de la propuesta de enseñanza de las áreas de lenguaje y matemáticas en la Institución Educativa San José del Pantano. Panorama, 9(17), 25-39. https://doi.org/10.15765/pnrm.v9i17.789

Freire, P. (2018). Pedagogía de la Autonomía. Buenos Aires, Argentina: Siglo XXI Editores.

García-Cabrero, B., Loredo, J., Carranza, G., Figueroa, A., Arbesú, I., Monroy, M., \& Reyes, R. (2008). Las aproximaciones teórico-metodológicas en los trabajos de la RIED: consideraciones en torno a la construcción de un modelo de evaluación de la práctica docente. La evaluación de los profesores como recurso para mejorar su práctica, 162-220.

Garza, R. (2018). Aprender cómo aprender. Ciudad de México: Editorial Trillas.

Hernández, V. (2017). Mapas conceptuales. La gestión del conocimiento en la didáctica. Ciudad de México, México: Alfaomega Grupo Editor S.A. de C.V.

Guzmán, J. P. H. (2015). El Estado en la sociedad, explicación de cómo la disposición de las burocracias determina los órdenes en países periféricos. Panorama, 9(17), 104-122. https://doi.org/10.15765/pnrm.v9i17.795

Hortal, A. (2018). Ética profesional y universidad. Caracas. Venezuela: Publicaciones UCAB.

León, E. M. (2014). Ampliación de horizontes epistemológicos para la construcción de nuevos entornos organizativos en instituciones educativas universitarias. Panorama, 8(14), 60-72. https://doi.org/10.15765/pnrm .v8i14.496

Martínez, M. (2012). La investigación cualitativa etnográfica en educación. Manual teórico-práctico. México: Editorial Trillas.

Montes, A. M. (2015). Optimización de la autoformación a través de la enseñanza virtual. Panorama, 9(17), 41-49. https://doi.org/10.15765/p nrm.v9i17.790

Olaves, V. (2018). Competencias del docente de educación integral en el contexto de la realidad educativa venezolana. Maracaibo, Venezuela: Colegio Universitario Dr. Rafael Belloso Chacín.

Pérez, M. (1994). Investigación cualitativa. Retos e interrogantes. España: Editorial la Muralla.

Rivera, G. O., \& Coronado, M. L. C. (2015). La formación científica en los primeros años de escolaridad. Panorama, 9(17), 10-23. https://doi.org/1 0.15765/pnrm.v9i17.788

Ruiz-Bolívar, C. (1998). La estrategia didáctica mediadora: ocho años después. Investigación y Postgrado 13(1), 15-38. 
Sandín, M. (2003). Investigación cualitativa en educación. Fundamentos y tradiciones. Madrid: McGraw Hill/interamericana, S.A.O.

Vargas, I. C. M., Pichardo, C. M. R., \& Íñiguez, F. J. M. (2015). Desarrollo de competencias integrales con tecnologías de la información y de la comunicación en educación superior a distancia. Panorama, 9(16), 9-19. https://doi.org/10.15765/pnrm.v9i16.631

Zavalza, M. (2006). Competencias docentes del profesorado universitario. Madrid: Narcea. 